\title{
The Fundamental and Rigidity Theorems for Pseudohermitian Submanifolds in the Heisenberg Groups
}

\author{
Hung-Lin Chiu* \\ Department of Mathematics, Tsing Hua University, Hsinchu 300, China.
}

Received October 31, 2019; Accepted March 3, 2020;

Published online January 22, 2021.

Dedicated to Professors Sun-Yung Alice Chang and Paul C. Yang on their 70th birthdays

\begin{abstract}
In this paper, we study some basic geometric properties of pseudohermitian submanifolds of the Heisenberg groups. In particular, we obtain the uniqueness and existence theorems, and some rigidity theorems.
\end{abstract}

AMS subject classifications: 32V05, 32V20, 32V30,53C56

Key words: Motion equations, structure equations, Darboux frame, Darboux derivative.

\section{Introduction}

In this paper, for $m \leq n$, we specify the ranges of indices as follows

$$
\begin{aligned}
& 1 \leq \alpha, \beta, \gamma, \sigma, \rho, \cdots \leq n, \quad 1 \leq j, k, l, \cdots \leq m \\
& m+1 \leq a, b, c, \cdots \leq n, \quad 1 \leq A, B, C, \cdots \leq 2 n
\end{aligned}
$$

\subsection{The Heisenberg groups}

The origin of pseudohermitian geometry came from the construction of a pseudohermitian connection, independently by N. Tanaka [15] and S. Webster [16]. In this paper, the Heisenberg group is a pseudohermitian manifold and it plays the role of the model in pseudohermitian geometry. That is, any pseudohermitian manifold with vanishing curvature and torsion locally is part of the Heisenberg group. Let $H_{n}$ be the Heisenberg group, with coordinates $\left(x_{\beta}, y_{\beta}, t\right)$. The group multiplication is defined by

$$
(x, y, t) \circ\left(x^{\prime}, y^{\prime}, t^{\prime}\right)=\left(x+x^{\prime}, y+y^{\prime}, t+t^{\prime}+y x^{\prime}-x y^{\prime}\right) .
$$

*Corresponding author. Email address: hlchiu@math.nthu.edu.tw (H. Chiu) 
The associated standard CR structure $J$ and contact form $\Theta$ are defined respectively by

$$
\begin{aligned}
J \stackrel{\circ}{e}_{\beta} & =\stackrel{\circ}{e}_{n+\beta}, \quad J \stackrel{\circ}{e}_{n+\beta}=-\stackrel{\circ}{e}_{\beta}, \\
\Theta & =d t+\sum_{\beta=1}^{n} x_{\beta} d y_{\beta}-y_{\beta} d x_{\beta},
\end{aligned}
$$

where

$$
\stackrel{e}{e}_{\beta}=\frac{\partial}{\partial x_{\beta}}+y_{\beta} \frac{\partial}{\partial t}, \stackrel{e}{e}_{n+\beta}=\frac{\partial}{\partial y_{\beta}}+x_{\beta} \frac{\partial}{\partial t} .
$$

The contact bundle is $\xi=\operatorname{ker} \Theta$. We refer the reader to $[2,3,5]$ for the details about the Heisenberg groups, and to $[6,11,12,15,16]$ for pseudohermitian geometry.

The symmetry group $P S H(n)$ of $H_{n}$ is the group consisting of all pseudohermitian transformations. Left translations $L_{p}$ are symmetries. Another kind of examples are a rotation $\Phi_{R}$ around the $t$-axis which is defined by

$$
\Phi_{R}\left(\begin{array}{c}
x \\
y \\
t
\end{array}\right)=\left(\begin{array}{cc}
R & 0 \\
0 & 1
\end{array}\right)\left(\begin{array}{l}
x \\
y \\
t
\end{array}\right)
$$

where $R=\left(\begin{array}{cc}A & -B \\ B & A\end{array}\right) \in S O(2 n)$. In [5], we showed that each symmetry $\Phi \in P S H(n)$ has the unique decomposition $\Phi=L_{p} \circ \Phi_{R}$, for some $p \in H_{n}$ and $R \in S O(2 n)$. Since the action of $\operatorname{PSH}(n)$ on $H_{n}$ is transitive, the associated geometry is a kind of Klein geometry. The corresponding Cartan geometry is just pseudohermitian geometry.

\subsection{Pseudohermitian submanifolds}

We now give the definition of pseudohermitian submanifold.

Definition 1.1. $A(2 m+1)$-dimensional pseudohermitian manifold $(M, \hat{J}, \hat{\theta})$ is called a pseudohermitian $^{\dagger}$ submanifold of $H_{n}, 1 \leq m \leq n$, if

- $\hat{\xi}=T M \cap \xi ;$

- $\hat{J}=\left.J\right|_{\hat{\xi}^{\prime}}$

- $\hat{\theta}=\left.\Theta\right|_{M}$

where $\hat{\xi}=k e r \hat{\theta}$ is the contact structure on $M$. The number $m$ is called the CR dimension of $M$.

Example 1.1. Suppose $M \hookrightarrow H_{n}$ is an embedded submanifold with CR dimension $n-1$. Then it is not hard to see that

${ }^{\dagger}$ In [6], S. Dragomir and G. Tomassini call it isopseudo-hermitian, instead of pseudohermitian. 
- In general, $\operatorname{dim}\left(T_{p} M \cap \xi_{p}\right) \geq 2 n-2$, for all $p \in M$.

- $\operatorname{dim}\left(T_{p} M \cap \xi_{p}\right)=2 n-2$, for a generic point $p \in M$.

All the generic points constitute the regular part of $M$, and those points $p$ such that $\operatorname{dim}\left(T_{p} M \cap \xi_{p}\right)=2 n-1$ are called the singular points. On the regular part $M_{r e}$, assume that $T_{p} M \cap \xi$ is invariant under $J$, then it inherits a pseudohermitian structure $(\hat{J}, \hat{\theta})$ from $H_{n}$ such that $\left(M_{r e}, \hat{J}, \hat{\theta}\right)$ is a pseudohermitian submanifold of $H_{n}$.

In Section 3, we define some local invariants for pseudohermitian submanifolds, including the second fundamental form, the normal connection and the fundamental vector field $v$ (see the definition after Proposition 3.1). In addition, from Proposition 3.1, we see that the fundamental vector field $v$ actually describes the difference between the two Reeb vector fields $T$ and $\hat{T}$, which are, respectively, associated with $H_{n}$ and the pseudohermitian submanifold $M$. Hence if $v=0$, then $\hat{T}=T$. That means that $T=\frac{\partial}{\partial t}$ is always tangent to $M$ at each point. Therefore, for such a submanifold, we call it vertical ${ }^{\ddagger}$. On the other hand, if $v \neq 0$ at each point, we call it completely non-vertical.

Example 1.2. The subspace $H_{m}=\left\{(z, t) \in H_{n} \mid z_{a}=0\right\} \subset H_{n}$ is a pseudohermitian submanifold of $H_{n}$. It is easy to see that $H_{m}$ is vertical.

Example 1.3. Let $S^{2 n-1} \subset H_{n}$ be the sphere defined by

$$
S^{2 n-1}=\left\{(z, 0) \in C^{n} \subset H_{n} \mid \sum_{\beta=1}^{n} z_{\beta} z_{\bar{\beta}}=1\right\} \text {. }
$$

There are two pseudohermitian structures induced on $S^{2 n-1}$, one is from $H_{n}$ and the other is from $C^{n}$. In Subsection 4.2, we show that these two induced pseudohermitian structures coincide. In addition, $S^{2 n-1}$ is completely non-vertical.

\subsection{Main theorems}

There are many literatures which were given for the problem about CR embeddability of CR manifolds into spheres $[4,9,10,17]$. In this paper, we obtain the fundamental theorems and rigidity theorems for pseudohermitian submanifolds in the Heisenberg groups. We have

Theorem 1.1 (Theorem A). The induced pseudohermitian structure, the second fundamental form, the normal connection, as well as the fundamental vector field constitute a complete set of invariants for pseudohermitian submanifolds of the Heisenberg groups.

\footnotetext{
‡In [6], S. Dragomir and G. Tomassini call it pseuo-Hermitian, instead of vertical pseudohermitian.
} 
Theorem A is shown in Section 5. It specifies that there are only four invariants for pseudohermitian submanifolds. That is, if two pseudohermitian submanifolds have the same such four invariants, then they are locally congruent with each other in the sense that they differ from each other by nothing more than an action of a symmetry.

Any pseudohermitian submanifold $M \subset H_{n}$ automatically satisfies a natural geometric condition which we call the integrability condition (defined in Subsection 5.2). Conversely, we will show that it is also a condition for an arbitrary pseudohermitian manifold to be (locally) embedded as a pseudohermitian submanifold of $H_{n}$.

Theorem 1.2 (Theorem B). Let $\left(M^{2 m+1}, J_{M}, \theta_{M}\right)$ be a simply connected pseudohermitian manifold satisfying the integrability condition for some $n \geq m$. Then $M$ can be embedded as a pseudohermitian submanifold of the Heisenberg group $H_{n}$.

Theorem B is shown in Section 6. In [9], S.-Y. Kim and J.-W. Oh also studied the problem of characterizing pseudohermitian manifolds which are pseudohermitian embeddable into the Heisenberg groups ${ }^{\S}$. In the case that $M$ is nondegenerate, S.-Y. Kim and J.-W. Oh used Cartan's prolongation method to show that the induced pseudohermitian structure constitutes a complete set of invariants. In addition, they gave a necessary and sufficient condition, in terms of Webster curvature and torsion tensor, for pseudohermitian manifolds to be embeddable into the Heisenberg groups nondegenerately. This condition is just equivalent to the integrability condition which we define in Subsection 5.2. However, S.-Y. Kim and J.-W. Oh did not deal with the degenerate cases.

In the case of CR codimension one, the nondegeneracy just means that the second fundamental form does not vanish at each point. In such a case, we basically recover the results of S.-Y. Kim and J.-W. Oh. Moreover, we give the rigidity theorems for pseudohermitian degenerate submanifolds, which are shown in Section 7 . We have

Theorem 1.3 (Theorem $C)$. Let $(M, \hat{J}, \hat{\theta})$ be a vertical, simply connected pseudohermitian submanifold of $H_{n}$ with $C R$ dimension $m=n-1$. Then we have

(i) if the second fundamental form II $\neq 0$ at each point, then the induced pseudohermitian structure $(\hat{J}, \hat{\theta})$ constitutes a complete set of invariants.

(ii) if $I I=0$, then $M$ is an open part of $H_{n-1}=\left\{z_{n}=0\right\}$, after a Heisenberg rigid motion.

Theorem 1.4 (Theorem D). Let $(M, \hat{J}, \hat{\theta})$ be a completely non-vertical, simply connected pseudohermitian submanifold of $H_{n}$ with $C R$ dimension $m=n-1$. Then we have

(i) the induced pseudohermitian structure $(\hat{J}, \hat{\theta})$ constitutes a complete set of invariants.

(ii) if the second fundamental form $I I=0$ (or, equivalently, the pseudohermitian torsion $A_{j k}=$ $0,1 \leq j, k \leq m)$, then $M$ is an open part of the standard sphere $S^{2 m+1} \subset H_{n}$, after a Heisenberg rigid motion.

Finally, in subsection 4.1, we study the general properties of vertical pseudohermitian submanifolds and obtain §In their paper, they used the pseudohermitian flat sphere as the ambient space, instead of the Heisenberg
group. But after a Cayley transformation, this two spaces are isomorphic as pseudohermitian manifolds 
Theorem 1.5 (Theorem E). Let $(M, \hat{\jmath}, \hat{\theta})$ be a vertical pseudohermitian submanifold of $H_{n}$. Then we have that the Webster-torsion vanishes and the Webster-Ricci tensor is non-positive, as well as the pseudohermitian connection and the tangential connection (see the definition in subsection 3.3) coincide.

For the fundamental theorems, we used Cartan's method of moving frame as well as calculus on Lie groups. And we prove (ii) of Theorem D by means of the motions equation of the Darboux frame. Therefore, in Section 2, we give a brief review of Cartan's method of moving frame, which includes the motion equations and the structure equations. We would like to end the introduction by pointing out that, in [4], Curry and Gover recently addressed the so called CR Bonnet theorem. They formulated and proved the theorem inspired by the conformal Bonnet theorem formulated and proved in terms of standard conformal tractors.

\section{Cartan's method of moving frame}

In this section, we give a brief review of Cartan's method of moving frame and Calculus on Lie groups. For the details, we refer the reader to [5]. Let $(X, G)$ be a Klein geometry. The philosophy of Elie Cartan is that in many cases, the symmetry group $G$ may be identified with a set of frame on $X$. Then to investigate the geometry of a submanifold $M$ of $X$, one associates the submanifold with a natural set of frames. In this situation, the infinitesimal motion of this natural frame should contain all the geometric information of the submanifold $M$. Now we will go along the idea of Elie Cartan to get a complete set of invariants for $M$.

\subsection{The frames on $H_{n}$}

An frame for $H_{n}$ is a set of vectors of the form

$$
\left(p ; e_{\beta}, e_{n+\beta}, T\right),
$$

where $p \in H_{n}, e_{\beta} \in \xi(p)$ and $e_{n+\beta}=J e_{\beta}$, for $1 \leq \beta \leq n$. In addition $\left\{e_{\beta}, e_{n+\beta}, T\right\}$ is an orthonormal frame with respect to the adapted metric $g_{\theta}$, which is defined by viewing the basis $\stackrel{\bullet}{\beta}_{\beta}, \stackrel{e}{e}_{n+\beta}, T$ as an orthonormal basis.

\subsection{Identifying $P S H(n)$ with a set of frames}

We identify a symmetry $\Phi$ with a frame $\left(p ; e_{\beta}, e_{n+\beta}, T\right)$, provided that $\Phi$ is the unique transformation on $H_{n}$ mapping the frame $\left(0 ; \stackrel{e}{\beta}_{\beta}, \stackrel{e}{e}_{n+\beta}, T\right)$ to the given frame $\left(p ; e_{\beta}, e_{n+\beta}, T\right)$. That is

$$
\Phi_{*}\left(0 ; \stackrel{\circ}{e}_{\beta}, \stackrel{\circ}{e}_{n+\beta}, T\right)=\left(\Phi(0) ; \Phi_{*} \stackrel{\stackrel{e}{e}}{\beta}, \Phi_{*} \stackrel{\circ}{e}_{n+\beta}, \Phi_{*} T\right)=\left(p ; e_{\beta}, e_{n+\beta}, T\right)
$$




\subsection{The matrix group representation of $P S H(n)$}

If we identify points of $H_{n}$ and $1 \times H_{n}$ by

$$
p \leftrightarrow\left(\begin{array}{c}
1 \\
p
\end{array}\right)
$$

hence a vector $X \in T H_{n}$ can be identified by

$$
X \leftrightarrow\left(\begin{array}{c}
0 \\
X
\end{array}\right)
$$

We thus identify $\Phi$ with a matrix $A \in G L(2 n+2, R)$ by

$$
\Phi \leftrightarrow\left(p ; e_{\beta}, e_{n+\beta}, T\right) \leftrightarrow A=\left(\begin{array}{cccc}
1 & 0 & 0 & 0 \\
p & e_{\beta} & e_{n+\beta} & T
\end{array}\right) .
$$

We have

$$
A\left(\begin{array}{l}
1 \\
q
\end{array}\right)=\left(\begin{array}{l}
1 \\
\tilde{q}
\end{array}\right), \quad \tilde{q}=\Phi(q)
$$

This shows that (2.1) gives a matrix group representation of $P S H(n)$.

\subsection{The motion equations}

Let $\omega$ be the (left) Maurer Cartan form of $\operatorname{PSH}(n)$. This is a $\operatorname{psh}(n)$-valued one form defined by

$$
\omega(v)=L_{g^{-1} *} v,
$$

for each $v \in T_{g} G$, where $G=P S H(n), g \in G$. That is, the Maurer Cartan form moves each vector $v$ to the identity element by the left translations. It is a natural way for us to identify each vector $v$ with a vector tangent to the identity. Since $\operatorname{PSH}(n)$ has a matrix group representation, The Maurer Cartan form has the simple elegant expression

$$
\omega=A^{-1} d A
$$

where $A \in \operatorname{PSH}(n)$ is the moving point. This formula (2.3) is equivalent to

$$
d A=A \omega
$$

which is called the motion equations of the Heisenberg group. Taking the exterior derivative of the motion equations, we get the structure equations

$$
d \omega+\omega \wedge \omega=0 .
$$




\subsection{The Darboux frames for pseudohermitian submanifolds}

Let $U \subset M$ be an open subset. For each point $p \in U$, we always choose the frame $\left\{Z_{\beta}, T\right\}$ such that $Z_{j} \in \hat{\xi}_{C}$ and $Z_{a} \in \hat{\xi}_{C}^{\perp}$, here $\hat{\xi}_{C}=\hat{\xi} \otimes C$ and $\hat{\xi}_{C}^{\perp}=\hat{\xi}^{\perp} \otimes C$. Such a moving frame $p \rightarrow\left(p ; Z_{\beta}, T\right)$ is called the Darboux frame (of complex version) over $U$.

Let $\left\{\theta^{\beta}, \theta\right\}$ be the dual of $\left\{Z_{\beta}, T\right\}$. Writing $Z_{\beta}=\frac{1}{2}\left(e_{\beta}-i e_{n+\beta}\right)$ and $\theta^{\beta}=\omega^{\beta}+i \omega^{n+\beta}$. Then $\left\{e_{A}, T\right\}$ and $\left\{\omega^{A}, \theta\right\}$ are dual to each other. This frame field $p \rightarrow\left(p ; e_{A}, T\right)$ is the real version of the Darboux frame. It is easy to see that $e_{k}, e_{n+k} \in \hat{\xi}, e_{a}, e_{n+a} \in \xi^{\perp}$, and $e_{n+\beta}=J e_{\beta}$.

Denoting $\hat{Z}_{j}=Z_{j}$ and writing $\hat{Z}_{j}=\frac{1}{2}\left(\hat{e}_{j}-i \hat{e}_{m+j}\right)$. Then we have $\hat{e}_{j}=e_{j}, \hat{e}_{m+j}=e_{n+j}$. Let $\left\{\hat{\omega}^{j}, \hat{\omega}^{m+j}, \hat{\theta}\right\}$ be the dual of $\left\{\hat{e}_{j}, \hat{e}_{m+j}, \hat{T}\right\}$. We also denote $\hat{\theta}^{j}=\hat{\omega}^{j}+i \hat{\omega}^{m+j}$.

\subsection{The Darboux derivative}

Let $f: U \rightarrow P S H(n)$ be a Darboux frame $f(p)=\left(p ; e_{A}, T\right)$. The Darboux derivative $\omega_{f}$ of $f$ is defined by

$$
\omega_{f}=\omega \circ f_{*}=f^{*} \omega .
$$

Therefore, it is just the usual differential $f_{*}$, provided that we have identified each vector with a vector tangent to the identity element by left translations. From (2.3),

$$
\omega_{f}=f^{*} \omega=f^{*}\left(A^{-1} d A\right)=(A \circ f)^{-1} d(A \circ f)=f^{-1} d f,
$$

or, equivalently

$$
d f=f \omega_{f} .
$$

This is the motion equations for the Darboux frame $f$. Again, taking the exterior derivative, we obtain the structure equations (the integrability condition)

$$
d \omega_{f}+\omega_{f} \wedge \omega_{f}=0
$$

Writing

$$
f(p)=\left(p ; e_{\beta}(p), e_{n+\beta}(p), T\right)
$$

and

$$
\omega_{f}=\left(\begin{array}{cccc}
0 & 0 & 0 & 0 \\
\omega^{\beta} & \omega_{\alpha}^{\beta} & \omega_{n+\alpha}^{\beta} & 0 \\
\omega^{n+\beta} & \omega_{\alpha}^{n+\beta} & \omega_{n+\alpha}{ }^{n+\beta} & 0 \\
\omega^{2 n+1} & \omega^{n+\alpha} & -\omega^{\alpha} & 0
\end{array}\right) .
$$

Since $\omega_{f}$ is a $p \operatorname{sh}(n)$-valued one form, the entry forms satisfy

$$
\begin{aligned}
& \omega_{a}^{b}=-\omega_{b}{ }^{a}, \quad \text { for } 1 \leq a, b \leq 2 n, \\
& \omega_{n+\alpha}{ }^{n+\beta}=\omega_{\alpha} \beta, \quad \omega_{\alpha}^{n+\beta}=-\omega_{n+\alpha}{ }^{\beta}, \quad \text { for } 1 \leq \alpha, \beta \leq n .
\end{aligned}
$$


Then the motion equations and structure equations, respectively, read

$$
\begin{aligned}
& d p=e_{\beta} \otimes \omega^{\beta}+e_{n+\beta} \otimes \omega^{n+\beta}+T \otimes \omega^{2 n+1}, \\
& d e_{\gamma}=e_{\beta} \otimes \omega_{\gamma}^{\beta}+e_{n+\beta} \otimes \omega_{\gamma}^{n+\beta}+T \otimes \omega^{n+\gamma}, \\
& d e_{n+\gamma}=e_{\beta} \otimes \omega_{n+\gamma}{ }^{\beta}+e_{n+\beta} \otimes \omega_{n+\gamma}{ }^{n+\beta}-T \otimes \omega^{\gamma}, \quad d T=0 ;
\end{aligned}
$$

and

$$
\begin{aligned}
& d \omega^{\beta}=-\omega_{\alpha}^{\beta} \wedge \omega^{\alpha}-\omega_{n+\alpha} \beta \wedge \omega^{n+\alpha}, \quad d \omega^{n+\beta}=-\omega_{\alpha}^{n+\beta} \wedge \omega^{\alpha}-\omega_{n+\alpha}{ }^{n+\beta} \wedge \omega^{n+\alpha}, \\
& d \omega^{2 n+1}=2 \sum_{\alpha=1}^{n} \omega^{\alpha} \wedge \omega^{n+\alpha}, \quad d \omega_{\alpha}^{\beta}=-\omega_{\gamma}^{\beta} \wedge \omega_{\alpha}^{\gamma}-\omega_{n+\gamma} \beta \wedge \omega_{\alpha}^{n+\gamma}, \\
& d \omega_{n+\alpha}{ }^{\beta}=-\omega_{\gamma}^{\beta} \wedge \omega_{n+\alpha}{ }^{\gamma}-\omega_{n+\gamma}{ }^{\beta} \wedge \omega_{n+\alpha}{ }^{n+\gamma} .
\end{aligned}
$$

\subsection{The complex version}

Writing

$$
F(p)=\left(p ; Z_{\beta}(p), T\right), \text { where } Z_{\beta}=\frac{1}{2}\left(e_{\beta}-i e_{n+\beta}\right),
$$

and

$$
\omega_{F}=\left(\begin{array}{ccc}
0 & 0 & 0 \\
\vartheta^{t} & \theta_{\gamma} \beta & 0 \\
\theta & i \bar{\vartheta} & 0
\end{array}\right),
$$

where $\vartheta=\left(\theta^{1}, \cdots, \theta^{n}\right), \theta^{\beta}=\omega^{\beta}+i \omega^{n+\beta}, \theta_{\gamma} \beta=\omega_{\gamma}^{\beta}+i \omega_{\gamma}^{n+\beta}$. And hence we have $\theta_{\gamma}{ }^{\beta}+\theta_{\bar{\beta}} \bar{\gamma}=0$. We have the complex version of motion equations

$$
d p=Z_{\beta} \otimes \theta^{\beta}+Z_{\bar{\beta}} \otimes \theta^{\bar{\beta}}+T \otimes \theta, \quad d Z_{\gamma}=Z_{\beta} \otimes \theta_{\gamma}{ }^{\beta}+\frac{1}{2} T \otimes i \theta^{\bar{\gamma}}, \quad d T=0 .
$$

And the structure equations are equivalent to

$$
d \omega_{F}+\omega_{F} \wedge \omega_{F}=0
$$

or

$$
d \theta^{\beta}=\theta^{\gamma} \wedge \theta_{\gamma}^{\beta}, \quad d \theta=i \theta^{\gamma} \wedge \theta^{\bar{\gamma}}, \quad d \theta_{\sigma}^{\beta}=\theta_{\sigma}^{\gamma} \wedge \theta_{\gamma}^{\beta} .
$$

\subsection{Calculus on Lie groups}

Let $M$ be a simply connected smooth manifold, $f: M \rightarrow P S H(n)$ be a smooth map. Recall that The (left) Darboux derivative $\omega_{f}$ of $f$ is the $p \operatorname{sh}(n)$-valued 1-form defined by $\omega_{f}=$ $\omega \circ f_{*}=f^{*} \omega$. The Darboux derivative plays an important role in the theory of calculus on Lie groups. The fundamental theorems are Theorem 2.1 and Theorem 2.2. 
Theorem 2.1 (The uniqueness theorem). Let $f_{1}, f_{2}: M \rightarrow P S H(n)$ be smooth maps. Then $\omega_{f_{1}}=\omega_{f_{2}}$ if and only if there exists $g \in P S H(n)$ such that $f_{2}(x)=g \cdot f_{1}(x)$ for all $x \in M$.

Theorem 2.1 says that two maps from $M$ into $P S H(n)$ are congruent to each other if and only if they have the same infinitesimal motions. Recall that $\omega_{f}$ satisfies the integrability conditions

$$
d \omega_{f}+\omega_{f} \wedge \omega_{f}=0
$$

Conversely, one has

Theorem 2.2 (The existence theorem). Let $\eta$ be a $p s h(n)$-valued one form on $M$ satisfying $d \eta+\eta \wedge \eta=0$. Then there is a smooth map $f: U \rightarrow P S H(n)$ such that $\left.\eta\right|_{U}=\omega_{f}$.

Theorem 2.2 totally depends on Frobenius Theorem. We will apply theorem 2.1 to the Darboux frames of pseudohermitian submanifolds. Then, to prove Theorem A, we are reduced to compute the Darboux derivatives of the Darboux frames. And using Theorem 2.2, we obtain Theorem B. For the details about calculus on Lie groups, we refer the reader to $[1,7,8,13,14]$.

\section{Local invariants of Pseudohermitian submanifolds}

In this section, we define some geometric invariants for pseudohermitian submanifolds.

\subsection{The fundamental vector field $v$}

Proposition 3.1. Let $(M, \hat{J}, \hat{\theta})$ be a pseudohermitian submanifold of $H_{n}$. Then there exists a unique horizontal vector field $v \in \hat{\zeta}^{\perp}$ such that $T+v \in T M$. Actually, denoting $\hat{T}=T+v$, it is not hard to see that $\hat{T}$ is the Reeb vector field associated to $\hat{\theta}$.

Proof. Let $\hat{T}=a T+\sum_{A=1} a^{A} e_{A}$, for some coefficients $a, a^{A}$. Since $1=\hat{\theta}(\hat{T})=\theta(\hat{T})=a$ and $\hat{T} \perp \hat{\xi}$, we have $\hat{T}=T+a^{a} e_{a}+a^{n+a} e_{n+a}$, and hence we can choose $v=a^{a} e_{a}+a^{n+a} e_{n+a}$. Next, suppose $\tilde{v} \in \hat{\xi}^{\perp}$ is another vector such that $T+\tilde{v} \in T M$. Then we have $v-\tilde{v} \in T M \cap \xi$, hence $v=\tilde{v}$.

We call $v$ in Proposition 3.1 the fundamental vector field.

- If $v \equiv 0$, then $\hat{T}=T$, and hence we call $M^{2 m+1}$ a vertical submanifold.

- If $v \neq 0$ at each point of $M$, then $M$ is completely non-vertical.

Proposition 3.2. We have

$$
\left.\omega^{j}\right|_{M}=\hat{\omega}^{j},\left.\omega^{n+j}\right|_{M}=\hat{\omega}^{m+j},\left.\omega^{a}\right|_{M}=\frac{1}{2}\left\langle v, e_{a}\right\rangle \hat{\theta},\left.\omega^{n+a}\right|_{M}=\frac{1}{2}\left\langle v, e_{n+a}\right\rangle \hat{\theta},
$$


where $\langle$,$\rangle is the Levi-metric, hence$

$$
\left.\theta^{j}\right|_{M}=\left.\hat{\theta}^{j} \quad \theta^{a}\right|_{M}=\left\langle v, Z_{a}\right\rangle \hat{\theta} .
$$

In particular, if $v=0$, then we have $\left.\theta^{a}\right|_{M}=0$.

Proof. We compute

$$
\omega^{j}(\hat{T})=\omega^{j}(T+v)=0, \quad \omega^{j}\left(\hat{e}_{k}\right)=\omega^{j}\left(e_{k}\right)=\delta_{j k}, \quad \omega^{j}\left(\hat{e}_{m+k}\right)=\omega^{j}\left(e_{n+k}\right)=0,
$$

and

$$
\begin{aligned}
& \omega^{n+j}(\hat{T})=\omega^{n+j}(T+v)=0, \quad \omega^{n+j}\left(\hat{e}_{k}\right)=\omega^{n+j}\left(e_{k}\right)=0, \\
& \omega^{n+j}\left(\hat{e}_{m+k}\right)=\omega^{n+j}\left(e_{n+k}\right)=\delta_{j k} .
\end{aligned}
$$

Therefore $\left\{\left.\omega^{j}\right|_{M},\left.\omega^{n+j}\right|_{M},\left.\theta\right|_{M}\right\}$ is the dual frame of $\left\{\hat{e}_{j}, \hat{e}_{m+j}, \hat{T}\right\}$. Similar computation shows that

$$
\left.\omega^{a}\right|_{M}=\frac{1}{2}\left\langle v, e_{a}\right\rangle \hat{\theta},\left.\quad \omega^{n+a}\right|_{M}=\frac{1}{2}\left\langle v, e_{n+a}\right\rangle \hat{\theta} .
$$

This completes the proof.

\subsection{The normal connection}

The normal connection $\nabla^{\perp}$ which is defined, on the normal complex bundle $\hat{\zeta}^{\perp} \otimes C$ spanned by $Z_{a}$, by

$$
\nabla^{\perp} Z_{a}=\theta_{a}^{b} \otimes Z_{b}
$$

which is the orthogonal projection of the pseudohermitian connection $\nabla Z_{a}$ onto the normal bundle.

\subsection{The tangential connection}

The tangential connection $\nabla^{t}$ which is defined, on the complex bundle $\hat{\xi}_{C}$ spanned by $Z_{j}$, by

$$
\nabla^{\perp} Z_{j}=\theta_{j}^{k} \otimes Z_{k}
$$

which is the orthogonal projection of the pseudohermitian connection $\nabla Z_{j}$ onto the contact bundle.

- Let $\hat{\theta}_{j}{ }^{k}$ be the pseudohermitian connection forms with respect to the frame field $Z_{j}$. Then, from (5.11), we have

$$
\left.\theta_{j}{ }^{k}\right|_{M}=\hat{\theta}_{j}^{k}+i \delta_{j k}|v|^{2} \hat{\theta} .
$$

Therefore, in general, $\nabla^{t} \neq \nabla^{p . h .}$, the associated pseudohermitian connection of $M$.

- If $M$ is vertical, then $\nabla^{t}=\nabla^{p \cdot h}$. 


\subsection{The second fundamental form}

Define the bilinear form $I I^{a}$ on $\hat{\xi}_{1,0}$ by

$$
I I^{a}(X, Y)=-\left\langle X, \nabla_{\bar{Y}} Z_{a}\right\rangle .
$$

- We have $I I^{a}=\theta^{j} \otimes \theta_{j}{ }^{a}$. If $\widetilde{Z}_{a}=C_{a}{ }^{b} Z_{b}$ is another normal frame field, then $\widetilde{I I}^{a}=C_{\bar{a}}{ }^{\bar{b}} I I^{b}$.

- $I I^{a} \otimes Z_{a}$ is independent of the choice of the normal frame field $Z_{a}$.

The second fundamental form II for $M$ is defined, to be a map

$$
I I: \hat{\xi}_{1,0} \times \hat{\xi}_{1,0} \rightarrow \hat{\xi}_{1,0}^{\perp}
$$

by

$$
I I=I I^{a} \otimes Z_{a}=\theta^{j} \otimes \theta_{j}^{a} \otimes Z_{a} .
$$

\section{General properties}

\subsection{Pseudohermitian submanifolds with $v \equiv 0$}

The canonical example is the Heisenberg subgroup $H_{m}$ which is defined by $H_{m}=\{(z, t) \in$ $\left.H_{n} \mid z_{a}=0\right\}$. Now we discuss the general properties of such kind of submanifolds. From Proposition 3.2, we have

$$
\left.\theta^{j}\right|_{M}=\hat{\theta}^{j}, \text { and }\left.\theta^{a}\right|_{M}=0 \text {. }
$$

Therefore, we have the structure equations

$$
\begin{aligned}
& d \theta^{j}=\theta^{k} \wedge \theta_{k}{ }^{j}, \quad 0=\theta^{k} \wedge \theta_{k}{ }^{a} \quad\left(\because \theta^{a}=0\right), \quad d \theta=i \theta^{k} \wedge \theta^{\bar{k}}, \\
& d \theta_{j}{ }^{l}=\theta_{j}{ }^{k} \wedge \theta_{k}{ }^{l}+\theta_{j}{ }^{c} \wedge \theta_{c}{ }^{l}, \quad d \theta_{j}{ }^{a}=\theta_{j}{ }^{k} \wedge \theta_{k}{ }^{a}+\theta_{j}{ }^{c} \wedge \theta_{c}{ }^{a}, \\
& d \theta_{a}{ }^{j}=\theta_{a}{ }^{k} \wedge \theta_{k}{ }^{j}+\theta_{a}{ }^{c} \wedge \theta_{c^{j}}{ }^{j}, \quad d \theta_{a}{ }^{b}=\theta_{a}{ }^{k} \wedge \theta_{k}{ }^{b}+\theta_{a}{ }^{c} \wedge \theta_{c}{ }^{b} .
\end{aligned}
$$

- From the first equation $d \theta^{j}=\theta^{k} \wedge \theta_{k}^{j}$ of (4.2), together with $\theta_{k}^{j}+\theta_{j}^{\bar{k}}$, we have

$$
\hat{\tau}^{j} \equiv 0, \hat{\theta}_{k}^{j}=\theta_{k}^{j},
$$

where $\hat{\tau}^{j}, \hat{\theta}_{k}{ }^{j}$ are the pseudohermitian torsion forms and connection forms with respect to the admissible coframe $\left\{\hat{\theta}^{j}\right\}$.

- From the second equation $0=\theta^{k} \wedge \theta_{k}^{a}$ of (4.2), together with Cartan lemma, we have

$$
\theta_{j}^{a}=h_{j k}^{a} \theta^{k},
$$

for some functions $h_{j k}^{a}$ satisfying $h_{j k}^{a}=h_{k j}^{a}$. Therefore

$$
I I=\theta^{j} \otimes \theta_{j}^{a} \otimes Z_{a}=h_{j k}^{a} \theta^{j} \otimes \theta^{k} \otimes Z_{a}
$$


- The fourth equation of (4.2)

$$
d \theta_{j}^{l}=\theta_{j}{ }^{k} \wedge \theta_{k}{ }^{l}+\theta_{j}^{c} \wedge \theta_{c}{ }^{l}
$$

is called the Gauss-like equation. Since $\theta_{j}{ }^{k}=\hat{\theta}_{j}^{k}$ and $\theta_{j}^{c}=h_{j k}^{c} \theta^{k}$, it is easy to see that the Gauss-like equation is equivalent to

$$
R_{j \bar{l} \zeta \bar{\eta}}=-\sum_{c} h_{j \zeta}^{c} h_{\bar{l} \bar{\eta}^{\prime}}^{\bar{c}}
$$

which implies $R_{\zeta \bar{\eta}}=-\sum_{c=m+1}^{n} h_{k \zeta}^{c} h_{\bar{k} \bar{\eta}^{\prime}}^{\bar{c}}$ and hence the Webster-Ricci tensor is nonpositive.

- The fifth equation of (4.2)

$$
d \theta_{j}^{a}=\theta_{j}{ }^{k} \wedge \theta_{k}{ }^{a}+\theta_{j}{ }^{c} \wedge \theta_{c}^{a}
$$

is equivalent to the sixth equation of (4.2)

$$
d \theta_{a}^{j}=\theta_{a}{ }^{k} \wedge \theta_{k}{ }^{j}+\theta_{a}{ }^{c} \wedge \theta_{c}{ }^{j} .
$$

Either one is called the Codazzi-like equation.

- The last equation of (4.2)

$$
d \theta_{a}^{b}=\theta_{a}{ }^{k} \wedge \theta_{k}^{b}+\theta_{a}^{c} \wedge \theta_{c}^{b}
$$

is called the Ricci-like equation.

\subsection{Pseudohermitian submanifolds with $v$ nowhere zero}

The canonical example is the standard sphere $S^{2 n-1} \subset C^{n} \subset H_{n}=C^{n} \times R, n \geq 2$. It is defined by

$$
S^{2 n-1}(r)=\left\{\left(z_{1}, \cdots, z_{n}, 0\right) \in H_{n} \mid \sum_{\beta=1}^{n} z_{\beta} z_{\bar{\beta}}=r^{2}\right\} .
$$

Let $L_{p}$ be a left translation, we compute the image of $(z, 0) \in S^{2 n-1}(r)$,

$$
L_{p}(z, 0)=p+x_{\beta} \stackrel{\varrho}{\beta}_{\beta}(p)+y_{\beta} \stackrel{\varrho}{n+\beta}_{n+1}(p),
$$

where $z_{\beta}=x_{\beta}+i y_{\beta}$, and hence the image of $S^{2 n-1}(r)$ under $L_{p}$ is

$$
L_{p}\left(S^{2 n-1}(r)\right)=\left\{q \in H_{n} \mid q-p \in \xi(p) \text {, and }|q-p|=r\right\},
$$

where the norm $|\cdot|$ is measured by the levi metric. Next, there are two pseudohermitian structures induced on $S^{2 n-1}$, one is from the Heisenberg group $H_{n}$, denoted by $(\hat{J}, \hat{\theta})$, and the other is from $C^{n}$. It is easy to see that these two induced pseudohermitian structures 
coincide on $S^{2 n-1}(r)$ as the following specifies. Let $u=\left(\sum_{\beta=1}^{n} z_{\beta} z_{\bar{\beta}}\right)-r^{2}$ be the defining function. We have

$$
\hat{\theta}=\left.\Theta\right|_{S^{2 n-1}}=x_{\beta} d y_{\beta}-y_{\beta} d x_{\beta}=\frac{i(\bar{\partial} u-\partial u)}{2},
$$

hence

$$
\hat{\xi}=\operatorname{ker} \hat{\theta}=T S^{2 n-1} \cap J_{C^{n}}\left(T S^{2 n-1}\right) \subset T C^{n},
$$

where $J_{C^{n}}$ is the standard complex structure on $C^{n}$.

Lemma 4.1. Let $p=(z, t) \in S^{2 n-1}$. If a vector $X=a_{\beta} \stackrel{\circ}{e}_{\beta}+a_{n+\beta} \stackrel{\circ}{e}_{n+\beta} \in \hat{\xi}(p)$, then $a_{\beta} y_{\beta}-a_{n+\beta} x_{\beta}=0$, where $z_{\beta}=x_{\beta}+i y_{\beta}$. In addition, we have

$$
X=a_{\beta} \stackrel{e}{\beta}_{\beta}+a_{n+\beta} \stackrel{\circ}{e}_{n+\beta}=a_{\beta} \frac{\partial}{\partial x_{\beta}}+a_{n+\beta} \frac{\partial}{\partial y_{\beta}},
$$

for all $X \in \hat{\zeta}$.

Proof. We compute

$$
X=a_{\beta} \stackrel{e}{e}_{\beta}+a_{n+\beta} \stackrel{e}{e}_{n+\beta}=a_{\beta} \frac{\partial}{\partial x_{\beta}}+a_{n+\beta} \frac{\partial}{\partial y_{\beta}}+\left(a_{\beta} y_{\beta}-a_{n+\beta} x_{\beta}\right) \frac{\partial}{\partial t} .
$$

Since $\hat{\xi} \subset T C^{n}$, we get $a_{\beta} y_{\beta}-a_{n+\beta} x_{\beta}=0$.

For all $X \in \hat{\xi}$,

$$
\begin{aligned}
\hat{J}(X) & =J\left(a_{\beta} \stackrel{\stackrel{e}{ }}{\beta}_{\beta}+a_{n+\beta} \stackrel{\stackrel{e}{e}}{n}+\beta\right)=a_{\beta} \stackrel{\bullet}{e}_{n+\beta}-a_{n+\beta} \stackrel{\circ}{e}_{\beta} \\
& =a_{\beta} \frac{\partial}{\partial y_{\beta}}-a_{n+\beta} \frac{\partial}{\partial x_{\beta}}=J_{C^{n}}\left(a_{\beta} \frac{\partial}{\partial x_{\beta}}+a_{n+\beta} \frac{\partial}{\partial y_{\beta}}\right) \\
& =J_{C^{n}}(X),
\end{aligned}
$$

which shows that $\hat{J}$ is also induced from $J_{C^{n}}$. On the other hand, from (4.12), we have

$$
\hat{T}=\frac{i\left(z_{\beta} \frac{\partial}{\partial z_{\beta}}-z_{\bar{\beta}} \frac{\partial}{\partial z_{\bar{\beta}}}\right)}{r^{2}}=\frac{\partial}{\partial t}+v,
$$

which implies that

$$
v=\frac{i\left(z_{\beta} \frac{\partial}{\partial z_{\beta}}-z_{\bar{\beta}} \frac{\partial}{\partial z_{\bar{\beta}}}\right)}{r^{2}}-\frac{\partial}{\partial t}=\frac{i\left(x_{\beta} \frac{\partial}{\partial y_{\beta}}-y_{\bar{\beta}} \frac{\partial}{\partial x_{\bar{\beta}}}\right)}{r^{2}}-\frac{\partial}{\partial t}=\frac{x_{\beta} \stackrel{\stackrel{e}{e}}{n+\beta}_{n}-y_{\beta} \stackrel{\leftrightarrow}{\beta}_{\beta}}{r^{2}} .
$$

This shows that the standard sphere $S^{2 n-1}(r)$ is completely non-vertical. 


\section{The uniqueness theorem}

In this section, we are going to prove Theorem A. Let $M$ and $N$ be two pseudohermitian submanifolds with the same CR dimension $m$. Suppose $\Phi$ is a Heisenberg rigid motion such that $\Phi(M)=N$ and denote $\varphi=\left.\Phi\right|_{M}$.

Let $\left\{Z_{\beta}\right\}$ be a frame field over $M$, and suppose $\widetilde{Z}_{\beta}=\Phi_{*} Z_{\beta}$, the set $\left\{\widetilde{Z}_{\beta}\right\}$ is a frame field over $N$. Suppose $\left\{\theta^{\beta}, \Theta\right\}$ and $\left\{\widetilde{\theta}^{\beta}, \Theta\right\}$ are the dual frame fields of $\left\{Z_{\beta}, T\right\}$ and $\left\{\widetilde{Z}_{\beta}, T\right\}$, respectively. Then we have

$$
\theta^{\beta}=\Phi^{*} \widetilde{\theta}^{\beta}, \quad \Theta=\Phi^{*} \Theta .
$$

In particular, we have

$$
\theta^{j}=\varphi^{*} \widetilde{\theta}^{j}, \quad \hat{\theta}=\varphi^{*} \widetilde{\theta},
$$

where $\hat{\theta}$ and $\widetilde{\theta}$ are the induced contact form on $M$ and $N$, respectively. (5.2) implies that $\varphi$ preserves the induced pseudohermitian structures.

From the structure equation on $H_{n}$, we compute

$$
\begin{aligned}
d \theta^{\beta} & =\theta^{\gamma} \wedge \theta_{\gamma}{ }^{\beta}=\left(\Phi^{*} \widetilde{\theta}^{\gamma}\right) \otimes \theta_{\gamma}{ }^{\beta} \\
\| & \\
d\left(\Phi^{*} \widetilde{\theta}^{\beta}\right) & =\Phi^{*}\left(d \widetilde{\theta}^{\beta}\right),
\end{aligned}
$$

which is equivalent to

$$
d \widetilde{\theta}^{\beta}=\widetilde{\theta}^{\gamma} \otimes\left(\Phi^{-1}\right)^{*} \theta_{\gamma}^{\beta}
$$

Together with

$$
\left(\Phi^{-1}\right)^{*} \theta_{\gamma}^{\beta}+\left(\Phi^{-1}\right)^{*} \theta_{\bar{\beta}}^{\bar{\gamma}}=\left(\Phi^{-1}\right)^{*}\left(\theta_{\gamma}{ }^{\beta}+\theta_{\bar{\beta}} \bar{\gamma}^{\bar{\gamma}}\right)=0,
$$

and by the uniqueness, we get

$$
\theta_{\gamma}^{\beta}=\Phi^{*} \widetilde{\theta}_{\gamma}^{\beta}
$$

In particular, we have

$$
\theta_{\gamma}^{\beta}=\varphi^{*} \widetilde{\theta}_{\gamma}^{\beta}
$$

and hence

$$
\langle I I, V\rangle=\varphi^{*}\left\langle\widetilde{I I}, \Phi_{*} V\right\rangle,
$$

for all $V \in \hat{\zeta}_{C}^{\perp}$.

The defferential $\Phi_{*}$ defines a vector bundle isomorphism

$$
\begin{array}{cccc}
\hat{\zeta}_{1,0}^{\perp} & \longrightarrow & \widetilde{\zeta}_{1,0}^{\perp} \\
\downarrow & & \downarrow \\
M & \longrightarrow & N,
\end{array}
$$

which preserving the hermitian structures induced from the levi-metric and cover $\varphi$, such that $\Phi_{*}$ preserves the normal connections, i.e.,

$$
\Phi_{*}\left(\nabla_{X}^{\perp} Z_{a}\right)=\widetilde{\nabla}_{\varphi_{*} X}^{\perp}\left(\Phi_{*} Z_{a}\right),
$$


for all $X \in T M$, where $\nabla^{\perp}$ and $\widetilde{\nabla}^{\perp}$ are the induced normal connections on $M$ and $N$, respectively. Finally, it is easy to see that $\Phi_{*} \nu=\widetilde{v}$.

Definition 5.1. Suppose that $M$ and $N$ are two pseudohermitian submanifolds of $H_{n}$ with the same $C R$ dimension $m$. We say that $M$ and $N$ have the same (induced) pseudohermitian structures, the second fundamental forms, the normal connections and the fundamental vector fields if there exists a vector bundle isomorphism $F: \hat{\xi}_{1,0}^{\perp} \rightarrow \widetilde{\xi}_{1,0}^{\perp}$, which preserves the induced hermitian structures and covers a map $\varphi: M \rightarrow N$, such that

- F preserves the induced pseudohermitian structures: $\varphi_{*} \circ \hat{J}=\widetilde{J} \circ \varphi_{*} ;$ and $\varphi^{*} \widetilde{\theta}=\hat{\theta}$;

- F preserves the second fundamental forms: $\langle I I, V\rangle_{\hat{\xi}_{1,0}^{\perp}}=\varphi^{*}\langle\widetilde{I I}, F V\rangle_{\widetilde{\xi}_{1,0}^{\perp}}$ for all $V \in \hat{\xi}_{1,0}^{\perp}$.

- F preserves the normal connections: $F\left(\nabla_{X}^{\perp} V\right)=\widetilde{\nabla}_{\varphi_{*} X}^{\perp}(F V)$, for all $X \in T M, V \in \hat{\xi}_{1,0}^{\perp}$.

- F preserves the fundamental vector field: $F v=\widetilde{v}$.

Therefore we conclude that if $M$ is congruent to $N$, then they have the same such four invariants. Conversely, we have

Theorem 5.1. Let $(M, \hat{J}, \hat{\theta})$ and $(N, \widetilde{J}, \widetilde{\theta})$ be two simply connected pseudohermitian submanifolds of $H_{n}$ with $C R$ dimension $m$. Suppose that they have the same (induced) pseudohermitian structures, the second fundamental forms, the normal connections and the fundamental vector fields. Then they differ by a Heisenberg rigid motion.

Corollary 5.1. If $M$ and $N$ are vertical, then the (induced) pseudohermitian structures, the second fundamental forms and the normal connections constitute a complete set of invariants.

\subsection{The proof of Theorem 5.1}

Let $(M, \hat{J}, \hat{\theta})$ be a pseudohermitian submanifold of $H_{n}$. Recall that we always choose the frame field $\left\{Z_{\beta}, T\right\}$ over $M$ such that $Z_{j} \in \hat{\xi}_{1,0}$ and $Z_{a} \in \hat{\xi}_{1,0}^{\perp}$. This is a Darboux frame. Let $\left\{\theta^{\beta}, \theta\right\}$ be the dual of $\left\{Z_{\beta}, T\right\}$. We would like to show that the restrictions of $\theta^{\beta}$ and $\theta_{\beta}^{\gamma}$ to $M$ are expressed as follows:

$$
\begin{aligned}
& \left.\theta^{j}\right|_{M}=\hat{\theta}^{j},\left.\quad \theta^{a}\right|_{M}=\left\langle v, Z_{a}\right\rangle \hat{\theta},\left.\quad \theta\right|_{M}=\hat{\theta}, \\
& \left.\theta_{j}{ }^{k}\right|_{M}=\hat{\theta}_{j}{ }^{k}+i \delta_{j k}|v|^{2} \hat{\theta},\left.\quad \theta_{j}{ }^{a}\right|_{M}=h_{j k}^{a} \hat{\theta}^{k}+i \delta_{j k}\left\langle v, Z_{a}\right\rangle \hat{\theta}^{k}+\left\langle\nabla_{\hat{Z}_{j}}^{\perp} v, Z_{a}\right\rangle \hat{\theta} ;
\end{aligned}
$$

and here $h_{j k}^{a}=I I^{a}\left(\hat{Z}_{j}, \hat{Z}_{k}\right)$, and $\left.\theta_{a}{ }^{b}\right|_{M}$ is the normal connection forms w.r.t. $\left\{Z_{a}\right\}$. This shows that the Darboux derivative of the Draboux frame is completely determined by the induced pseudohermitian structure, the second fundamental form, the normal connection and the fundamental vector field.

Now we prove (5.11).

$$
d \theta^{a}=\theta^{j} \wedge \theta_{j}{ }^{a}+\theta^{b} \wedge \theta_{b}{ }^{a}=\hat{\theta}^{j} \wedge \theta_{j}{ }^{a}+\left\langle\nu, Z_{b}\right\rangle \hat{\theta} \wedge \theta_{b}{ }^{a} .
$$


On the other hand,

$$
\begin{aligned}
d \theta^{a} & =d\left(\left\langle v, Z_{a}\right\rangle \hat{\theta}\right)=d\left\langle v, Z_{a}\right\rangle \wedge \hat{\theta}+\left\langle v, Z_{a}\right\rangle d \hat{\theta} \\
& =\left(\left\langle\nabla v, Z_{a}\right\rangle+\left\langle v, \nabla-Z_{a}\right\rangle\right) \wedge \hat{\theta}+\left\langle v, Z_{a}\right\rangle d \hat{\theta} \\
& =\left(\left\langle\nabla^{\perp} v, Z_{a}\right\rangle+\left\langle v, \nabla^{\perp} Z_{a}\right\rangle\right) \wedge \hat{\theta}+\left\langle v, Z_{a}\right\rangle d \hat{\theta},
\end{aligned}
$$

and

$$
\left\langle v, \nabla \div Z_{a}\right\rangle=\left\langle v, \theta_{a}{ }^{b}(\bar{\bullet}) \otimes Z_{b}\right\rangle=\left\langle v, Z_{b}\right\rangle \theta_{\bar{a}}{ }^{\bar{b}} .
$$

From (5.12)-(5.14), we obtain

$$
\hat{\theta}^{j} \wedge \theta_{j}{ }^{a}=\left\langle\nabla^{\perp} v, Z_{a}\right\rangle \wedge \hat{\theta}+\left\langle v, Z_{a}\right\rangle d \hat{\theta} .
$$

That is,

$$
\begin{aligned}
& \theta_{j}{ }^{a}\left(\hat{Z}_{k}\right) \hat{\theta}^{j} \wedge \hat{\theta}^{k}+\theta_{j}{ }^{a}\left(\hat{Z}_{\bar{k}}\right) \hat{\theta}^{j} \wedge \hat{\theta}^{\bar{k}}+\theta_{j}{ }^{a}(\hat{T}) \hat{\theta}^{j} \wedge \hat{\theta} \\
= & \left\langle\nabla_{\hat{Z}_{k}}^{\perp} v, Z_{a}\right\rangle \hat{\theta}^{k} \wedge \hat{\theta}+\left\langle\nabla_{\hat{Z}_{\bar{k}}}^{\perp} v, Z_{a}\right\rangle \hat{\theta}^{\bar{k}} \wedge \hat{\theta}+i\left\langle v, Z_{a}\right\rangle \hat{\theta}^{j} \wedge \hat{\theta}^{\bar{j}},
\end{aligned}
$$

which implies

$$
\begin{array}{ll}
\theta_{j}{ }^{a}(\hat{T})=\left\langle\nabla_{\hat{Z}_{j}}^{\perp} v, Z_{a}\right\rangle, & 0=\left\langle\nabla \hat{\bar{Z}}_{j} v, Z_{a}\right\rangle, \\
\theta_{j}{ }^{a}\left(\hat{Z}_{\bar{k}}\right)=i \delta_{j k}\left\langle v, Z_{a}\right\rangle, & \theta_{j}{ }^{a}\left(\hat{Z}_{k}\right)=\theta_{k}{ }^{a}\left(\hat{Z}_{j}\right)=h_{j k}^{a},
\end{array}
$$

and thus

$$
\theta_{j}^{a}=h_{j k}^{a} \hat{\theta}^{k}+i \delta_{j k}\left\langle\nu, Z_{a}\right\rangle \hat{\theta}^{\bar{k}}+\left\langle\nabla \frac{\grave{Z}_{j}}{\nu}, Z_{a}\right\rangle \hat{\theta} .
$$

Now we compute

$$
d \theta^{k}=\theta^{j} \wedge \theta_{j}{ }^{k}+\theta^{a} \wedge \theta_{a}{ }^{k}=\hat{\theta}^{j} \wedge \theta_{j}{ }^{k}+\hat{\theta} \wedge\left(\left\langle\nu, Z_{a}\right\rangle \theta_{a}{ }^{k}\right) .
$$

On the other hand,

$$
d \theta^{k}=d \hat{\theta}^{k}=\hat{\theta}^{j} \wedge \hat{\theta}_{j}^{k}+\hat{\theta} \wedge \tau^{k} .
$$

By Cartan lemma, there exists functions $B_{j l}^{k}, B_{j(m+1)}^{k}, B_{(m+1) l}^{k}$ and $B_{(m+1)(m+1)}^{k}$ such that

$$
\hat{\theta}_{j}^{k}=\theta_{j}^{k}+B_{j l}^{k} \hat{\theta}^{l}+B_{j(m+1)}^{k} \hat{\theta}, \quad \hat{\tau}^{k}=\left(\left\langle v, Z_{a}\right\rangle \theta_{a}{ }^{k}\right)+B_{(m+1) l}^{k} \hat{\theta}^{l}+B_{(m+1)(m+1)}^{k} \hat{\theta},
$$

where $B_{j l}^{k}=B_{l j}^{k}$ and $B_{j(m+1)}^{k}=B_{(m+1) j}^{k}$, for $1 \leq j, k, l \leq m$. Since $\hat{\tau}^{k}=A_{\hat{l}^{k}}^{k} \hat{\theta}^{\bar{l}}$, comparing with (5.21), we get

$$
\begin{aligned}
& A^{k}{ }_{\bar{l}}=\sum_{a=m+1}^{n}\left\langle v, Z_{a}\right\rangle \theta_{a}{ }^{k}\left(\hat{Z}_{\bar{l}}\right)=-\sum_{a=m+1}^{n}\left\langle\nu, Z_{a}\right\rangle h_{\bar{k} \bar{l}}^{\bar{a}} \\
& B_{(m+1) l}^{k}=-\sum_{a=m+1}^{n}\left\langle v, Z_{a}\right\rangle \theta_{a}{ }^{k}\left(\hat{Z}_{l}\right)=-i \delta_{k l}|v|^{2}, \\
& B_{(m+1)(m+1)}^{k}=-\sum_{a=m+1}^{n}\left\langle\nu, Z_{a}\right\rangle \theta_{a}{ }^{k}(\hat{T})=\sum_{a=m+1}^{n}\left\langle\nu, Z_{a}\right\rangle\left\langle\nabla_{\hat{Z}_{\bar{k}}}^{\perp} \nu, Z_{\bar{a}}\right\rangle .
\end{aligned}
$$


Finally, since $\theta_{j}{ }^{k}+\theta_{\bar{k}} \bar{j}=0$ and $\hat{\theta}_{j}{ }^{k}+\hat{\theta}_{\bar{k}}{ }^{\bar{j}}=0$, we have, from (5.21), $B_{j l}^{k}=0$, and hence

$$
\hat{\theta}_{j}^{k}=\theta_{j}^{k}-i \delta_{j k}|v|^{2} \hat{\theta} .
$$

This completes the proof of (5.11).

\subsection{The integrability condition}

Let $(M, \hat{\jmath}, \hat{\theta})$ be a pseudohermitian submanifold of $H_{n}$. We choose a Darboux frame $\left\{Z_{\beta}, T\right\}$ over $M$. Let $\left\{\theta^{\beta}, \theta\right\}$ be the dual of $\left\{Z_{\beta}, T\right\}$.

Definition 5.2. The restriction to $M$ of the structure equations of $H_{n}$,

$$
d \theta^{\beta}=\theta^{\gamma} \wedge \theta_{\gamma}^{\beta}, \quad d \theta=i \theta^{\gamma} \wedge \theta^{\bar{\gamma}}, \quad d \theta_{\sigma}^{\beta}=\theta_{\sigma}^{\gamma} \wedge \theta_{\gamma}{ }^{\beta},
$$

is defined to be the integrability condition of $M$. Note that the restrictions of $\theta^{\beta}$ and $\theta_{\beta}^{\gamma}$ to $M$ have the expressions of the forms as (5.11) specifies..

\section{The existence theorem}

In this section, we would like to show Theorem B. Let $\left(M, J_{M}, \theta_{M}\right)$ be a pseudohermitian manifold with CR dimension $m$. Since the existence theory is local, we assume that $M$ is simply connected. Putting $\xi_{M}=\operatorname{ker} \theta_{M}$ and $\eta=\theta_{M}$.

- Let $\xi_{M}^{\perp}$ be a complex vector bundle over $M$, of complex dimension $n-m$, with a Hermitian metric $h_{M}^{\perp}$ and a connection $\nabla^{M}$ compatible with $h_{M}^{\perp}$.

- Suppose $\left\{W_{1}, \cdots, W_{m} \in T_{1,0} M\right\}$ is an orthonormal CR holomorphic frame field of $M$. Its dual is denoted by $\left\{\eta^{1}, \cdots, \eta^{m}\right\}$. Let $\hat{\eta}_{j}{ }^{k}$ be the pseudohermitian conection forms w.r.t. $W_{j}$. We have $\hat{\eta}_{j}^{k}+\hat{\eta}_{\bar{k}}{ }^{\bar{j}}=0$.

- Suppose $\left\{W_{m+1}, \cdots, W_{n}\right\}$ is an orthonormal frame field of $\xi_{M}^{\perp}$ w.r.t. $h_{M}^{\perp}$ and $\eta_{a}{ }^{b}$ are the connection forms w.r.t. $\left\{W_{a}\right\}$, i.e.,

$$
\nabla^{M} W_{a}=\eta_{a}^{b} \otimes W_{b}
$$

We have $\eta_{a}^{b}+\eta_{\bar{b}}^{\bar{a}}=0$.

- Let $I I_{M}: T_{1,0} M \times T_{1,0} M \rightarrow \xi_{M}^{\perp}$ be a $\xi_{M}^{\perp}$-valued complex bilinear form.

- Let $\mu$ be a real section of the bundle $\xi_{M}^{\perp}$ over $M$. And define

$$
\eta^{a}=\left\langle\mu, W_{a}\right\rangle \eta, \quad \eta_{j}{ }^{k}=\hat{\eta}_{j}{ }^{k}+i \delta_{j k}|\mu|^{2} \eta, \quad \eta_{j}^{a}=g_{j k}^{a} \eta^{k}+i \delta_{j k}\left\langle\mu, W_{a}\right\rangle \eta^{\bar{k}}+\left\langle\nabla_{W_{j}}^{M} \mu, W_{a}\right\rangle \eta,
$$

where $\langle\rangle=,h_{M}^{\perp}$ and $g_{j k}^{a}=\left\langle I I_{M}\left(W_{j}, W_{k}\right), W_{a}\right\rangle$. 
- Finally, define $\eta_{a}{ }^{j}$ by $\eta_{j}{ }^{a}+\eta_{\bar{a}}{ }^{j}=0$.

Theorem 6.1. Suppose that the pseudohermitian manifold $\left(M^{2 m+1}, J_{M}, \theta_{M}\right)$, together with $I I_{M}$, the compatible connection $\nabla^{M}$ and the section $\mu$ satisfies the integrability condition, in the sense that $\eta^{\beta}, \eta$ and $\eta_{\gamma}^{\beta}$ satisfy (5.24). Then

- There exists an embedding $\phi$ such that $\left(M, J_{M}, \theta_{M}\right)$ can be embedded into $H_{n}$ with $C R$ dimensionn $m$.

- In addition, there exists a vector bundle isomorphism $\Psi: \xi_{M}^{\perp} \rightarrow \hat{\xi}_{1,0}^{\perp}$, covering $\phi$, such that $\Psi^{*} I I=I I_{M}, \Psi^{*} \nabla^{\perp}=\nabla^{M}$, and $\Psi^{*} v=\mu$, where $\hat{\xi}_{1,0}^{\perp}, I I, \nabla^{\perp}$ and $v$ are, respectively, the induced normal bundle, second fundamental form, normal connection and fundamental vector field $v$ over $\phi(M)$.

Proof. Let $b=\left(\eta^{1}, \cdots, \eta^{n}\right), \bar{b}=\left(\eta^{\overline{1}}, \cdots, \eta^{\bar{n}}\right)$. Define the matrix $\Pi$ by

$$
\Pi=\left(\begin{array}{ccc}
0 & 0 & 0 \\
b^{t} & \eta_{\gamma}^{\beta} & 0 \\
\eta & i \bar{b} & 0
\end{array}\right) .
$$

The integrability condition means that

$$
d \Pi+\Pi \wedge \Pi=0 .
$$

Taking the real version $\zeta$ of $\Pi$,

$$
\zeta=\left(\begin{array}{cccc}
0 & 0 & 0 & 0 \\
\lambda^{\beta} & \lambda_{\alpha}^{\beta} & \lambda_{n+\alpha^{\beta}} & 0 \\
\lambda^{n+\beta} & \lambda_{\alpha}^{n+\beta} & \lambda_{n+\alpha}{ }^{n+\beta} & 0 \\
\lambda & \lambda^{n+\alpha} & -\lambda^{\alpha} & 0
\end{array}\right),
$$

where $\lambda=\eta, \eta^{\beta}=\lambda^{\beta}+i \lambda^{n+\beta}$ and $\eta_{\gamma}{ }^{\beta}=\lambda_{\gamma}{ }^{\beta}+i \lambda_{\gamma}{ }^{n+\beta}$. Then $\eta_{\gamma}{ }^{\beta}+\eta_{\bar{\beta}} \bar{\gamma}=0$ implies that $\zeta$ is a $p \operatorname{sh}(n)$-valued one form. And (6.4) is equivalent to $d \zeta+\zeta \wedge \zeta=0$. Therefore, by calculus on Lie groups, we have that $\zeta$ is the Darboux derivative of some map $f: M \rightarrow \operatorname{PSH}(n)$, that is,

$$
\zeta=f^{*} \omega .
$$

Define a map $\phi: M \rightarrow H_{n}$ by $\phi=\pi \circ f$, where $\pi$ is the bundle projection $\pi: P S H(n) \rightarrow H_{n}$, and define a bundle map $\Psi: \xi_{M}^{\perp} \rightarrow \hat{\zeta}_{1,0}^{\perp}$ by $\Psi\left(p, W_{a}\right)=\left(\phi(p), Z_{a}\right)$. Then, using (6.5), it is easy to check that $\Psi$ and $\phi$ satisfy all what we want. This completes the proof.

\section{Rigidity theorems for submanifolds with CR co-dimension one}

In this section, we prove some rigidity theorems for pseudohermitian submanifolds, including both the nondegenerate and degenerate cases. 
Theorem 7.1. Let $(M, \hat{J}, \hat{\theta})$ be a vertical, simply connected pseudohermitian submanifold of $H_{n}$ with $C R$ dimension $m=n-1$. Suppose that the second fundamental form $I I=0$.Then $M$ is an open subset $U$ of $H_{n-1}=\left\{z_{n}=0\right\}$ after a Heisenberg rigid motion.

Proof. In the case $m=n-1$, we write $\theta_{j}^{n}=h_{j k} \theta^{k}$, here $h_{j k}$ are the coefficients of the second fundamental form $I I$. If $I I=0$, then $\theta_{j}^{n}=0$. On the other hand, $v=0$ implies $\theta^{n}=0$. Hence the structure equations of $H_{n}$, restricting to $M$, are reduced to

$$
d \theta^{j}=\theta^{k} \wedge \theta_{k}{ }^{j}, \quad d \theta=i \theta^{k} \wedge \theta^{\bar{k}}, \quad d \theta_{j}{ }^{l}=\theta_{j}{ }^{k} \wedge \theta_{k}{ }^{l}, \quad d \theta_{n}{ }^{n}=0 .
$$

The last equation of (7.1) says that $\theta_{n}{ }^{n}$ is closed, and hence locally is exact. By the transformation law of the normal connection, we can choose a normal frame $Z_{n}$ such that the corresponding connection form $\theta_{n}{ }^{n}$ vanishes. On the other hand, the first three equations of (7.1) is just the structure equations of $H_{n-1}$. This means that $M$ is an open part $U$ of $H_{n-1} \subset H_{n}$, up to a pseudohermitian transformatio $\varphi$ from $M$ to $U$. Define $F$ by $F\left(x, Z_{n}(x)\right)=\left(\varphi(x), \stackrel{\circ}{Z}_{n}\right)$. Then $F$ defines the normal bundle isomorphism covering $\varphi$ which preserving the induced pseudohermitian structures, the second fundamental forms and the normal connections of $M$ and $U$, respectively. Hence $\varphi$ is just the restriction of a Heisenberg rigid motion.

For a vertical pseudohermitian submanifold of $H_{n}$, we define a flat point of $M$ to be a point such that $I I=0$ at that point. Theorem 7.4 says that the induced pseudohermitian structure is the only invariant for vertical pseudohermitian submanifolds without flat points.

Theorem 7.2. Let $(M, \hat{J}, \hat{\theta})$ and $(N, \widetilde{J}, \widetilde{\theta})$ be two vertical, simply connected pseudohermitian submanifolds of $H_{n}$ without flat points. Suppose both of them are of $C R$ dimension $m=n-1$. If there exists a pseudohermitian transformation $\phi: M \rightarrow N$, then $\phi=\left.\Phi\right|_{M}$ for some Heisenberg rigid motion $\Phi$.

Proof. By Theorem 5.1, it suffices to show that both the second fundamental form and the normal connection are completely determined by the induced pseudohermitian structure. We write $\theta_{j}{ }^{n}=h_{j k} \theta^{k}$. Then, from the Gauss-like equation, we have

$$
d \theta_{j}^{l}-\theta_{j}^{k} \wedge \theta_{k}^{l}=-h_{j p} h_{\overline{\bar{q}}} \theta^{p} \wedge \theta^{\bar{q}}
$$

On the other hand

$$
d \theta_{j}{ }^{l}-\theta_{j}{ }^{k} \wedge \theta_{k}{ }^{l}=d \hat{\theta}_{j}{ }^{l}-\hat{\theta}_{j}{ }^{k} \wedge \hat{\theta}_{k}{ }^{l}=R_{j}{ }^{l} p \bar{q} \theta^{p} \wedge \theta^{\bar{q}} .
$$

From (7.2) and (7.3), we see that the Gauss-like equation is equivalent to

$$
R_{j \bar{l} p \bar{q}}=-h_{j p} h_{\bar{l} \bar{q}}
$$

which implies that

$$
R_{j \bar{l} p \bar{q}}=0 \Leftrightarrow I I=0 .
$$


If $I I \neq 0$, then there exists $h_{j k} \neq 0$ for some $j, k$. Since $I I$ is a symmetric bilinear form, after a frame transformation, we can assume, w.l.o.g., that $h_{11} \neq 0$. Then we have

$$
R_{j \overline{1} p \overline{1}}=-h_{j p} h_{\overline{1} \overline{1}} .
$$

In particular,

$$
h_{11}=\sqrt{-R_{1 \overline{1} 1 \overline{1}}} e^{i \varphi} \text {, for some } \varphi .
$$

On the other hand, if we take another orthonormal frame field $\left\{\tilde{Z}_{\beta}\right\}$ such that

$$
\tilde{Z}_{j}=Z_{j}, \quad \tilde{Z}_{n}=e^{i \psi} Z_{n},
$$

for some $\psi$. Then we have the transformation law for connection forms

$$
\tilde{\theta}_{j}{ }^{k}=\theta_{j}{ }^{k}, \quad \tilde{\theta}_{j}{ }^{n}=e^{-i \psi} \theta_{j}{ }^{n}, \quad \tilde{\theta}_{n}{ }^{j}=e^{i \psi} \theta_{n}{ }^{j}, \quad \tilde{\theta}_{n}{ }^{n}=\theta_{n}{ }^{n}+i d \psi .
$$

Notice that $\theta_{j}^{n}=h_{j k} \theta^{k}, \tilde{\theta}_{j}^{n}=\tilde{h}_{j k} \tilde{\theta}^{k}$ and $\tilde{\theta}^{k}=\theta^{k}$, hence we immediately have

$$
\tilde{h}_{j k}=e^{-i \psi} h_{j k}, \text { for all } j, k .
$$

In particular, $\tilde{h}_{11}=e^{-i \psi} h_{11}=e^{i(\varphi-\psi)} \sqrt{-R_{1 \overline{1}} \overline{1}}$. Taking $\psi=\varphi$, we have

$$
\tilde{h}_{11}=\sqrt{-R_{1 \overline{1} 1 \overline{1}}}=\sqrt{-\tilde{R}_{1 \overline{1} 1 \overline{1}}} \text {. }
$$

Formula (7.11) means that we can always choose a frame field $\left\{Z_{\beta}\right\}$ such that $h_{11}=$ $\sqrt{-R_{1 \overline{1}} 1 \overline{1}}$, and hence

$$
h_{j k}=-\frac{R_{j \overline{1} k \overline{1}}}{\sqrt{-R_{1 \overline{1} 1 \overline{1}}}}, \text { for all } j, k \text {. }
$$

This means that the second fundamental form II is completely determined by the induced pseudohermitian structure.

We proceed to show that the normal connection is also completely determined by the induced pseudohermitian structure. For each $j$,

$$
d \theta_{j}^{n}=d\left(h_{j k} \theta^{k}\right)=\left(d h_{j k}-h_{j l} \theta_{k}^{l}\right) \wedge \theta^{k} .
$$

On the other hand,

$$
d \theta_{j}{ }^{n}=\theta_{j}{ }^{k} \wedge \theta_{k}{ }^{n}+\theta_{j}{ }^{n} \wedge \theta_{n}{ }^{n}=\left(h_{l k} \theta_{j}{ }^{l}-h_{j k} \theta_{n}{ }^{n}\right) \wedge \theta^{k} .
$$

From (7.13) and (7.14), we have, for each $j, k$,

$$
d h_{j k}-h_{j l} \theta_{k}{ }^{l}-h_{l k} \theta_{j}{ }^{l}+h_{j k} \theta_{n}{ }^{n}=\sum_{l=1}^{n-1} B_{j k l} \theta^{l},
$$


for some $B_{j k l}$, which satisfying $B_{j k l}=B_{j l k}$. In particular

$$
h_{11} \theta_{n}{ }^{n}=-\left(d h_{11}-h_{1 l} \theta_{1}^{l}-h_{l 1} \theta_{1}^{l}\right)+\sum_{l=1}^{n-1} B_{11 l} \theta^{l} .
$$

The conjugate of (7.16) is,

$$
-h_{11} \theta_{n}{ }^{n}=h_{\overline{1} \overline{1}} \theta_{\bar{n}}^{\bar{n}}=-\left(d h_{\overline{1} \overline{1}}-h_{\overline{1} \bar{l}} \theta_{\overline{1}}^{\bar{l}}-h_{\overline{1} \overline{1}} \theta_{\overline{1}}^{\bar{l}}\right)+\sum_{l=1}^{n-1} B_{\overline{1} \bar{l} \bar{l}} l^{\bar{l}} .
$$

Taking the sum of (7.16) and (7.17)

$$
\sum_{l=1}^{n-1} B_{11 l} \theta^{l}+\sum_{l=1}^{n-1} B_{\overline{1} \overline{1} \bar{l}} \theta^{\bar{l}}=\left(h_{11, l} \theta^{l}+h_{11, \bar{l}} \bar{\theta}^{\bar{l}}+h_{11,0} \theta\right)+\text { conjugate }
$$

which implies that $B_{11 l}=h_{11, l}+h_{\overline{1} \overline{1}, l}$. Substituting this into (7.16), we get

$$
\theta_{n}^{n}=\frac{h_{\overline{1} \overline{1}, l^{l}} l^{l} h_{11, \bar{l}} \theta^{\bar{l}}-h_{11,0} \theta}{h_{11}},
$$

which means that $\theta_{n}{ }^{n}$ is completely determined by the induced pseudohermitian structure.

Remark 7.1. (i) From (7.18), we also get $h_{11,0}+h_{\overline{1} \overline{1}, 0}=0$. Therefore, in the case $n=2$, we have $T h_{11}=0$ or $T R=R_{0}=0$.

(ii) Also, for $n=2$, we have that $\theta_{2}^{2}=2 \theta_{1}{ }^{1}-d\left(\ln h_{11}\right)+2 Z_{1}\left(\ln h_{11}\right) \theta^{1}$.

Theorem 7.3. Let $(M, \hat{J}, \hat{\theta})$ and $(N, \widetilde{J}, \widetilde{\theta})$ be two simply connected pseudohermitian submanifolds of $H_{n}$ with $C R$ dimension $m=n-1$. Suppose, in addition, that their fundamental vector fields are nowhere zero. If they have the same (induced) pseudohermitian structures. Then they locally differ by a Heisenberg rigid motion. More explicitly, if there exists a pseudohermitian transformation $\phi: M \rightarrow N$, then $\phi=\left.\Phi\right|_{M}$ for some Heisenberg rigid motion $\Phi$.

Proof. The key point is that if $v(p) \neq 0$ for each point $p \in M$, then we can always choose a Darboux frame $p \rightarrow\left(p ; e_{\beta}, J e_{\beta}, T\right)$ such that

$$
e_{n}=-\frac{v}{|v|}, e_{2 n}=J e_{n} .
$$

Then we would like to compute the Darboux derivative of the Barboux frame. It is equivalent to computing the restrictions of $\theta^{\beta}, \theta_{\gamma}^{\beta}$ to $M$. To finish the proof, we need to show the Darboux derivative is completely determined by the induced pseudohermitian structure.

$$
d \theta^{\beta}=\theta^{\gamma} \wedge \theta_{\gamma}{ }^{\beta}=\hat{\theta}^{k} \wedge \theta_{k}{ }^{\beta}+\theta^{n} \wedge \theta_{n}{ }^{\beta}=\hat{\theta}^{k} \wedge \theta_{k}{ }^{\beta}+\hat{\theta} \wedge\left(-|v| \theta_{n}{ }^{\beta}\right) .
$$


On the other hand,

$$
d \theta^{j}=d \hat{\theta}^{j}=\hat{\theta}^{k} \wedge \hat{\theta}_{k}^{j}+\hat{\theta} \wedge \hat{\tau}^{j},
$$

and

$$
d \theta^{n}=-d(|v| \hat{\theta})=\hat{\theta}^{k} \wedge\left(-i|v| \hat{\theta}^{\bar{k}}\right)+\hat{\theta} \wedge(d|v|) .
$$

From (7.21), (7.22) and (7.23), there exists complex-valued functions $a_{\beta \gamma}^{j}$ such that $a_{\beta \gamma}^{j}=$ $a_{\gamma \beta}^{j}$ and

$$
\theta_{k}^{j}=\hat{\theta}_{k}^{j}+a_{k l}^{j} \hat{\theta}^{l}+a_{k n}^{j} \hat{\theta}, \quad-|v| \theta_{n}^{j}=\hat{\tau}^{j}+a_{n l}^{j} \hat{\theta}^{l}+a_{n n}^{j} \hat{\theta},
$$

Also, there exists complex-valued functions $b_{\beta \gamma}$ such that $b_{\beta \gamma}=b_{\gamma \beta}$ and

$$
\theta_{k}^{n}=-i|v| \hat{\theta}^{\bar{k}}+b_{k l} \hat{\theta}^{l}+b_{k n} \hat{\theta}, \quad-|v| \theta_{n}^{n}=d|v|+b_{n l} \hat{\theta}^{l}+b_{n n} \hat{\theta} .
$$

From (7.24),

$$
0=\theta_{k}^{j}+\theta_{j}^{\bar{k}}=\left(\hat{\theta}_{k}^{j}+\hat{\theta}_{\bar{j}}^{\bar{k}}\right)+a_{k l}^{j} \hat{\theta}^{l}+a_{\bar{j} l}^{\bar{k}} \hat{\theta}^{\bar{l}}+\left(a_{k n}^{j}+a_{j \bar{n}}^{\bar{k}}\right) \hat{\theta},
$$

hence

$$
a_{k l}^{j}=0, a_{k n}^{j}+a_{j \bar{n}}^{\bar{k}}=0, \text { for all } 1 \leq j, k, l \leq m .
$$

Similarly, and notice that we write $\hat{\tau}^{j}=A^{j} \hat{k}^{\hat{k}}$, we have

$$
\begin{aligned}
& b_{n l}=-2\left(\hat{Z}_{l}|v|\right), \quad b_{n n}+b_{\bar{n} \bar{n}}=-2(\hat{T}|v|), \quad a_{n l}^{j}=i \delta_{j l}|v|^{2}, \\
& b_{j l}=\frac{A^{\bar{j}}}{|v|}, \quad a_{n n}^{j}=|v| b_{\bar{j} \bar{n}}=-2|v|\left(\hat{Z}_{\bar{j}}|v|\right),
\end{aligned}
$$

for all $1 \leq j, l \leq m$. From (7.24), (7.27), (7.28), we have, for all $1 \leq j, k \leq m$,

$$
\theta_{k}^{j}=\hat{\theta}_{k}^{j}+\left(i \delta_{j k}|v|^{2}\right) \hat{\theta}, \quad-|v| \theta_{n}^{j}=\hat{\tau}^{j}+\left(i \delta_{j l}|v|^{2}\right) \hat{\theta}^{l}-2|v|\left(\hat{Z}_{j}|v|\right) \hat{\theta} .
$$

From (7.25), (7.28),

$$
\theta_{k}{ }^{n}=-i|v| \hat{\theta}^{\bar{k}}+\frac{A^{\bar{k}_{l}}}{|v|} \hat{\theta}^{l}-2\left(\hat{Z}_{k}|v|\right) \hat{\theta}, \quad-|v| \theta_{n}{ }^{n}=(d|v|)-2\left(\hat{Z}_{l}|v|\right) \hat{\theta}^{l}+b_{n n} \hat{\theta} .
$$

From the look of (7.29) and (7.30), there is only one term $b_{n n}$ not determined yet. In order to complete the proof, we need to show that both $b_{n n}$ and $|v|$ are completely determined by the induced pseudohermitian structure. For this, using (7.29) and (7.30), we compute

$$
\begin{aligned}
d \theta_{k}{ }^{j}= & \theta_{k}{ }^{l} \wedge \theta_{l}{ }^{j}+\theta_{k}{ }^{n} \wedge \theta_{n}{ }^{j} \\
= & \left(\hat{\theta}_{k}{ }^{l}+\left(i \delta_{l k}|v|^{2}\right) \hat{\theta}\right) \wedge\left(\hat{\theta}_{l}{ }^{j}+\left(i \delta_{j l}|v|^{2}\right) \hat{\theta}\right) \\
& +\frac{1}{|v|^{2}}\left(i|v|^{2} \hat{\theta}^{\bar{k}}-A^{\bar{k}_{l}} \hat{\theta}^{l}+2|v|\left(\hat{Z}_{k}|v|\right) \hat{\theta}\right) \wedge\left(A^{j}{ }_{i} \hat{\theta}^{\bar{l}}+i|v|^{2} \hat{\theta}^{j}-2|v|\left(\hat{Z}_{\bar{j}}|v|\right) \hat{\theta}\right),
\end{aligned}
$$


that is,

$$
\begin{aligned}
& d \theta_{k}{ }^{j}-\hat{\theta}_{k}{ }^{l} \wedge \hat{\theta}_{l}{ }^{j} \\
= & \frac{1}{|v|^{2}}\left(-i A^{\bar{k}}{ }_{l}|v|^{2} \hat{\theta}^{l} \wedge \hat{\theta}^{j}-i A^{j}|v|^{2} \hat{\theta}^{\bar{l}} \wedge \hat{\theta}^{\bar{k}}+\left(-A^{\bar{k}_{l}} A^{j}{ }_{\bar{q}}+\delta_{j}^{l} \delta_{k}^{q}|v|^{4}\right) \hat{\theta}^{l} \wedge \hat{\theta}^{\bar{q}}+\left(2 A^{\bar{k}}{ }_{l}|v|\left(\hat{Z}_{\bar{j}}|v|\right)\right.\right. \\
& \left.\left.2 i \delta_{j}^{l}|v|^{3}\left(\hat{Z}_{k}|v|\right)\right) \hat{\theta}^{l} \wedge \hat{\theta}+\left(-2 A^{j}{ }_{\bar{q}}|v|\left(\hat{Z}_{k}|v|\right)-2 i \delta_{k}^{q}|v|^{3}\left(\hat{Z}_{\bar{j}}|v|\right)\right) \hat{\theta}^{\bar{q}} \wedge \hat{\theta}\right) .
\end{aligned}
$$

On the other hand, from (7.29) and using the structure equations of the pseudohermitian structure, we have

$$
\begin{aligned}
d \theta_{k}{ }^{j}-\hat{\theta}_{k}{ }^{l} \wedge \hat{\theta}_{l}{ }^{j}= & d \hat{\theta}_{k}{ }^{j}-\hat{\theta}_{k}{ }^{l} \wedge \hat{\theta}_{l}^{j}+d\left(i \delta_{j k}|v|^{2} \hat{\theta}\right) \\
= & R_{k}{ }^{j} p \bar{q} \hat{\theta}^{p} \wedge \hat{\theta}^{\bar{q}}+W_{k}{ }^{j} \hat{\theta}^{p} \wedge \hat{\theta}-W^{j}{ }_{k \bar{p}} \hat{\theta}^{\bar{p}} \wedge \hat{\theta}+i \hat{\theta}_{k} \wedge \hat{\tau}^{j}-\hat{\tau}_{k} \wedge \hat{\theta}^{j} \\
& +i \delta_{j k}\left(\left(\hat{Z}_{l}|v|^{2}\right) \hat{\theta}^{l} \wedge \hat{\theta}+\left(\hat{Z}_{\bar{l}}|v|^{2}\right) \hat{\theta}^{\bar{l}} \wedge \hat{\theta}\right)-\delta_{j k}|v|^{2} \hat{\theta}^{l} \wedge \hat{\theta}^{\bar{l}} .
\end{aligned}
$$

Comparing the coefficients of the same terms in (7.31) and (7.32), and notice that $\hat{\tau}^{j}=$ $A^{j} \bar{k}^{\bar{\theta}}$, we get

$$
\begin{aligned}
& R_{k}{ }^{j}{ }_{l \bar{q}}-\delta_{j k} \delta_{l q}|v|^{2}=-\frac{A^{\bar{k}}{ }_{l} A^{j} \bar{q}}{|v|^{2}}+\delta_{j}^{l} \delta_{k}^{q}|v|^{2}, \\
& W_{k}{ }^{j}+i \delta_{j k}\left(\hat{Z}_{l}|v|^{2}\right)=2 \frac{A^{\bar{k}}{ }_{l}}{|v|}\left(\hat{Z}_{\bar{j}}|v|\right)-2 i \delta_{j}^{l}|v|\left(\hat{Z}_{k}|v|\right), \\
& -W^{j}{ }_{k \bar{l}}+i \delta_{j k}\left(\hat{Z}_{\bar{l}}|v|^{2}\right) x=-2 \frac{A^{j} \bar{l}}{|v|}\left(\hat{Z}_{k}|v|\right)-2 i \delta_{k}^{l}|v|\left(\hat{Z}_{\bar{j}}|v|\right),
\end{aligned}
$$

for all $1 \leq j, k, l, q \leq m$. From the first equation of (7.33),

$$
\begin{aligned}
R_{k \bar{j}} & =R_{k \bar{j} l \bar{l}}=-\sum_{l=1}^{m} \frac{A^{\bar{k}}{ }_{l} A_{\bar{l}}^{j}}{|v|^{2}}+\sum_{l=1}^{m}\left(\delta_{j k} \delta_{l l}+\delta_{j}^{l} \delta_{k}^{l}\right)|v|^{2} \\
& =\left\{\begin{array}{l}
-\sum_{l=1}^{m} \frac{A^{\bar{k}} A_{l}^{j_{\bar{l}}}}{|v|^{2}}, \text { for } k \neq j \\
-\left(\sum_{l=1}^{m} \frac{A^{\bar{k}} A^{j} \bar{j}_{\bar{l}}}{|v|^{2}}\right)+(m+1)|v|^{2}, \quad \text { for } k=j .
\end{array}\right.
\end{aligned}
$$

In particlar

$$
R=R_{k \bar{k}}=-\frac{|A|^{2}}{|v|^{2}}+m(m+1)|v|^{2} .
$$

Formula (7.35) is equivalent to

$$
|v|^{2}=\frac{R+\sqrt{R^{2}+4 m(m+1)|A|^{2}}}{2 m(m+1)} .
$$


Finally, we would like to compute $b_{n n}$. From (7.28), we see that $b_{n n}+b_{\bar{n} \bar{n}}=-2(\hat{T}|v|)$, i.e., $b_{n n}=(-\hat{T}|v|)+i\left(\operatorname{Im} b_{n n}\right)$. So we only compute $\operatorname{Im} b_{n n}$. For this, using (7.29) and (7.30)

$$
\begin{aligned}
d \theta_{j}{ }^{n}= & \theta_{j}{ }^{k} \wedge \theta_{k}{ }^{n}+\theta_{j}{ }^{n} \wedge \theta_{n}{ }^{n}=\left(\theta_{j}{ }^{k}-\delta_{j}^{k} \theta_{n}{ }^{n}\right) \wedge \theta_{k}{ }^{n} \\
= & \frac{1}{|v|^{2}}\left(|v| \theta_{j}{ }^{k}+\delta_{j}^{k}\left(-|v| \theta_{n}{ }^{n}\right)\right) \wedge\left(|v| \theta_{k}{ }^{n}\right) \\
= & \frac{1}{|v|^{2}}\left(|v| \hat{\theta}_{j}{ }^{k}+\delta_{j}^{k}\left[-\left(\hat{Z}_{l}|v|\right) \hat{\theta}^{l}+\left(\hat{Z}_{\bar{l}}|v|\right) \hat{\theta}^{\bar{l}}+i\left(|v|^{3}+\operatorname{Im} b_{n n}\right) \hat{\theta}\right]\right) \\
& \wedge\left(-i|v|^{2} \hat{\theta}^{\bar{k}}+A^{\bar{k}} \hat{\theta}^{l}-2|v|\left(\hat{Z}_{k}|v|\right) \hat{\theta}\right) \\
= & -i|v| \hat{\theta}_{j}{ }^{k} \wedge \hat{\theta}^{l}+\hat{\theta}_{j}{ }^{k} \wedge\left(\frac{A^{\bar{k}} l}{|v|} \hat{\theta}-2\left(\hat{Z}_{k}\right) \hat{\theta}\right)+\frac{1}{|v|^{2}}\left(-\left(\hat{Z}_{l}|v|\right) \hat{\theta}^{l}+\left(\hat{Z}_{\bar{l}}|v|\right) \hat{\theta}^{\bar{l}}\right. \\
& \left.\quad+i\left(|v|^{3}+\operatorname{Im} b_{n n}\right) \hat{\theta}\right) \wedge\left(-i|v|^{2} \hat{\theta}^{\bar{j}}+A^{\bar{j}}{ }_{l} \hat{\theta}^{l}-2|v|\left(\hat{Z}_{j}|v|\right) \hat{\theta}\right) .
\end{aligned}
$$

On the other hand, using (7.30) and the structure equations of the pseudohermitian structure,

$$
\begin{aligned}
d \theta_{j}{ }^{n} & =d\left(i|v| \hat{\theta}^{\bar{j}}+\frac{A^{\bar{j}} l}{|v|} \hat{\theta}^{l}-2\left(\hat{Z}_{j}|v|\right) \hat{\theta}\right) \\
& =-i|v| \hat{\theta}_{j}^{k} \wedge \hat{\theta}^{\bar{k}}+i|v| \hat{\tau}^{\bar{j}} \wedge \hat{\theta}-i(d|v|) \wedge \hat{\theta}^{\bar{j}} \\
& -2 d\left(\hat{Z}_{j}|v|\right) \wedge \hat{\theta}-2\left(\hat{Z}_{j}|v|\right) d \hat{\theta}+d\left(\frac{A^{\bar{j}_{l}}}{|v|}\right) \wedge \hat{\theta}^{l}+\left(\frac{A^{\bar{j}_{l}}}{|v|}\right) d \hat{\theta}^{l} .
\end{aligned}
$$

For each $j, 1 \leq j \leq m$, comparing the coefficients of the terms in (7.37) and (7.38), we get

$$
2\left(\hat{Z}_{\bar{j}}\left(\hat{Z}_{j}|v|\right)-\hat{\theta}_{j}^{k}\left(\hat{Z}_{\bar{j}}\right)\left(\hat{Z}_{k}|v|\right)\right)-i(\hat{T}|v|)=2 \frac{\left|\hat{Z}_{j}\right| v||^{2}}{|v|}+|v|^{3}+\operatorname{Im} b_{n n}-\frac{\sum_{l=1}^{m}\left|A_{j l}\right|^{2}}{|v|} .
$$

Writing

$$
|v|_{j \bar{j}}=\hat{Z}_{\bar{j}}\left(\hat{Z}_{j}|v|\right)-\hat{\theta}_{j}^{k}\left(\hat{Z}_{\bar{j}}\right)\left(\hat{Z}_{k}|v|\right),
$$

and taking the sum for $j$ over 1 to $m$, we have

$$
-\hat{\Delta}_{b}|v|=-\bar{\square}_{b}|v|-i m(\hat{T}|v|)=2 \frac{\left|\hat{\partial}_{b}\right| v||^{2}}{|v|}+m\left(|v|^{3}+\operatorname{Im} b_{n n}\right)-\frac{|A|^{2}}{|v|},
$$

where $\square_{b}$ and $\hat{\partial}_{b}$ are the Kohn Laplacian and $\partial_{b}$-operator on $M^{2 m+1}$. Hence $b_{n n}$ is determined. Substituting (7.39) into (7.30), we get

$$
|v| \theta_{n}{ }^{n}=\hat{\partial}_{b}|v|-\overline{\hat{\partial}}_{b}|v|+i\left(\frac{\hat{\Delta}_{b}|v|}{m}+|v|^{3}+\frac{\left(2\left|\hat{\partial}_{b}\right| v||^{2}-|A|^{2}\right)}{m|v|}\right) \hat{\theta} .
$$

This completes the proof. 
Theorem 7.3 says that for pseudohermitian submanifolds of CR dimension $m=n-$ 1 , in which there is no zero for $v$, the induced pseudohermitian structure constitute a complete set of invariant. Moreover, we have that if the pseudohermitian torsion of $M$ vanishes, then $M$ locally is part of the standard sphere as Theorem 7.3 describes.

Theorem 7.4. Let $(M, \hat{J}, \hat{\theta})$ be a simply connected pseudohermitian submanifold with $C R$ dimension $m=n-1$. Suppose that the fundamental vector fields is nowhere zero. If $A_{\beta \gamma} \equiv 0$, then the Webster curvature $R$ is constant, hence it is part of the standard sphere after a Heisenberg rigid motion.

Proof. Suppose that $A_{\beta \gamma}=0$. From (7.33) and (7.35), we get $R=m(m+1)|v|^{2}=$ constant. Next we claim

$$
\omega_{a}^{2 n}=-|v| \omega^{a}, \text { for } a=1, \cdots, 2 n-1 .
$$

From (7.30), we have for $1 \leq k \leq n-1$,

$$
\theta_{k}^{n}=-i|v| \hat{\theta}^{\bar{k}}=-i v\left|\theta^{\bar{k}}=-i\right| v \mid\left(\omega^{k}-i \omega^{n+k}\right) .
$$

And from (7.40),

$$
\theta_{n}^{n}=i|v|^{2} \hat{\theta}=-i v\left|\theta^{n}=-i\right| v \mid\left(\omega^{n}+i \omega^{2 n}\right) .
$$

On the other hand, we see that for $1 \leq k \leq n$, we have

$$
\theta_{k}^{n}=\omega_{k}^{n}+i \omega_{k}^{2 n}=\omega_{n+k}^{2 n}+i \omega_{k}^{2 n} .
$$

Comparing (7.42)-(7.44), we get the claim (7.41). In addition, we also have

$$
\omega^{2 n}=0, \omega^{n}=-|v| \theta .
$$

Substituting (7.41) and (7.45) into the motion equation

$$
\begin{aligned}
d e_{2 n} & =e_{\beta} \otimes \omega_{2 n}{ }^{\beta}+e_{n+\beta} \otimes \omega_{2 n}{ }^{n+\beta}-T \otimes \omega^{n} \\
& =\sum_{\beta=1}^{n-1} e_{\beta} \otimes\left(|v| \omega^{\beta}\right)+e_{n} \otimes\left(|v| \omega^{n}\right)+\sum_{\beta=1}^{n-1} e_{n+\beta} \otimes\left(|v| \omega^{n+\beta}\right)+T \otimes(|v| \theta) .
\end{aligned}
$$

That is

$$
d\left(\frac{e_{2 n}}{|v|}\right)=e_{A} \otimes \omega^{A}+T \otimes \theta=d X, \quad \text { on } \mathrm{M} .
$$

We conclude that on $M$

$$
X-X^{0}=\frac{e_{2 n}}{|v|}, \quad \text { for some } X^{0} \in H_{n} \text {. }
$$

Writing

$$
\begin{aligned}
\frac{e_{2 n}}{|v|} & =a_{A}(X) \stackrel{\ominus}{e}_{A}(X), \quad \text { for some coefficient functions } a_{A}, \\
X & =\left(X_{1}, \cdots, X_{2 n}, X_{2 n+1}\right), \quad X^{0}=\left(X_{1}^{0}, \cdots, X_{2 n}^{0}, X_{2 n+1}^{0}\right) .
\end{aligned}
$$


From (7.48), we have

$$
a_{A}(X)=X_{A}-X_{A}^{0}, \quad A=1, \cdots, 2 n,
$$

and hence

$$
\begin{aligned}
X-X^{0} & =\frac{e_{2 n}}{|v|}=a_{A}(X) \stackrel{e}{A}_{A}(X) \\
& =\left(X_{\beta}-X_{\beta}^{0}\right)\left(\frac{\partial}{\partial x_{\beta}}+X_{n+\beta} \frac{\partial}{\partial t}\right)+\left(X_{n+\beta}-X_{n+\beta}^{0}\right)\left(\frac{\partial}{\partial y_{\beta}}-X_{\beta} \frac{\partial}{\partial t}\right) \\
& =\left(X_{\beta}-X_{\beta}^{0}\right)\left(\frac{\partial}{\partial x_{\beta}}+X_{n+\beta}^{0} \frac{\partial}{\partial t}\right)+\left(X_{n+\beta}-X_{n+\beta}^{0}\right)\left(\frac{\partial}{\partial y_{\beta}}-X_{\beta}^{0} \frac{\partial}{\partial t}\right) \\
& =a_{A}(X) \stackrel{e}{A}_{A}\left(X^{0}\right),
\end{aligned}
$$

with $\sum_{A=1}^{2 n} a_{A}^{2}=\frac{1}{|v|^{2}}$. This completes the proof.

Theorem 7.4 is the same as Theorem 7.5.

Theorem 7.5. Let $(M, \hat{J}, \hat{\theta})$ be a simply connected pseudohermitian submanifold with $C R$ dimension $m=n-1$. Suppose that the fundamental vector fields is nowhere zero. If $I I=0$, then the Webster curvature $R$ is constant, hence it is part of the standard sphere after a Heisenberg rigid motion.

Proof. Note that in the proof of Theorem 7.1, we choose a Darboux frame such that

$$
e_{n}=-\frac{v}{|v|} \text {. }
$$

This implies that $\left\langle v, Z_{n}\right\rangle=-|v|$. Hence, from (5.11), we have

$$
\theta_{j}{ }^{n}=h_{j k} \hat{\theta}^{k}-i|v| \theta^{\bar{j}}, \bmod \hat{\theta} .
$$

Comparing with (7.30), we get

$$
h_{j k}=\frac{A_{j k}}{|v|}, \quad 1 \leq j, k \leq m .
$$

Therefore

$$
A_{j k}=0 \Leftrightarrow I I=0 .
$$

This complete the proof.

\section{Acknowledgments}

The author's research is supported in part by NCTS and in part by MOST 106-2115-M007-017-MY3. He would like to thank Prof. Jih-Hsin Cheng, Prof. Jenn-Fang Hwang, and Prof. Paul Yang for regular kind encouragement and advising in his research. 


\section{References}

[1] S. S. Chern, W. H. Chen and K. S. Lam, Lecture on Differential Geometry, World Scientific, 1981.

[2] O. Calin, D. C. Chang and P. Greiner, Geometric analysis on the Heisenberg group and its generalizations, AMS/IP Studies in Advanced Mathematics, 40(2007).

[3] O. Calin and D. C. Chang, Sub-Riemannian Geometry: General Theory and Examples, Cambridge, New York: Cambridge University Press, 2009.

[4] S. N. Curry and A. R. Gover, CR embedded submanifolds of CR manifolds, Memo. Am. Math. Soc., 258 (2019), 1-81.

[5] H. L. Chiu and S. H. Lai, The fundamental theorem for hypersurfaces in Heisenberg groups, Calc. Var. Partial Differential Equations, 54(1) (2015), 1091-1118.

[6] S. Dargamir and G. Tomassini, Differential geometry and analysis on CR manifolds, Progress Math., 246 (2006).

[7] P. Griffiths, On Cartan's Method of Lie groups and moving frames as applied to uniqueness and existence questions in differential geometry, Duke Math. J., 41 (1974), 775-814.

[8] T. A. Ivey, and J. M. Landsberg, Cartan for beginners: differential geometry via moving frames and exterior differential systems, Graduate Studies Math., 61 (2003).

[9] S. Y. Kim, and J. Q. Oh, Local embeddability of pseudohermitian manifolds into spheres, Math. Ann., 334 (2006), 783-807.

[10] L. Lempert, Imbedding Cauchy-Riemann manifolds into a sphere, Int. J. Math., 1 (1990), 91-108.

[11] J. M. Lee, The Fefferman metric and pseudohermitian invariants, Trans. Am. Math. Soc., 296 (1986), 411-429.

[12] J. M. Lee, Pseudo-Einstein structures on CR manifolds, Am. J. Math., 110 (1988), 157-178.

[13] R. S. Palais and C. L. Terng, Critical point theory and submanifold geometry, Lecture Notes on Differential Geometry, 7 (1988), 280p.

[14] R. W. Sharp, Differential geometry Cartan's generalization of Klein's Erlangen program, Graduate Texts in Math., 61 (1997), 426p.

[15] N. Tanaka, A Differential Geometric Study on Strongly Pseudo-convex Manifolds, Kinokuniya Company Ltd., Tokyo, 1975.

[16] S. M. Webster, Pseudo-Hermitian structures on a real Hypersurface, J. Diff. Geom., 13 (1978), 25-41.

[17] S. M. Webster, The rigidity of CR-hypersurfaces in a sphere, Indiana Univ Math. J., 28(3) (1979), 405-416. 